\title{
Stage IB Breast Cancer AJCC v7
}

National Cancer Institute

\section{Source}

National Cancer Institute. Stage IB Breast Cancer A/CC v7. NCI Thesaurus. Code C85836.

Stage IB includes: (T0, N1 mi, M0); (T1, N1 mi, M0). T0: No evidence of primary tumor. T1:

Tumor $20 \mathrm{~mm}$ or less in greatest dimension. T1 includes T1mi. T1 mi: T umor $1 \mathrm{~mm}$ or less in greatest dimension. N1mi: Nodal micrometastases. M0: No clinical or radiographic evidence of distant metastasis. $\mathrm{MO}$ includes $\mathrm{MO}(\mathrm{i}+$ ). (AJCC 7th Ed.) 